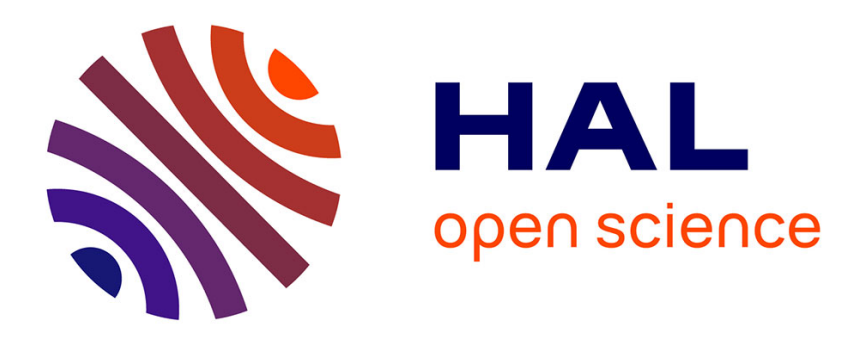

\title{
Modal coupling in one-dimensional electromechanical structured continua
}

Stefano Vidoli, Francesco Dell'Isola

\section{To cite this version:}

Stefano Vidoli, Francesco Dell'Isola. Modal coupling in one-dimensional electromechanical structured continua. Acta Mechanica, 2000, pp.14. hal-00501520

\section{HAL Id: hal-00501520 \\ https://hal.science/hal-00501520}

Submitted on 12 Jul 2010

HAL is a multi-disciplinary open access archive for the deposit and dissemination of scientific research documents, whether they are published or not. The documents may come from teaching and research institutions in France or abroad, or from public or private research centers.
L'archive ouverte pluridisciplinaire HAL, est destinée au dépôt et à la diffusion de documents scientifiques de niveau recherche, publiés ou non, émanant des établissements d'enseignement et de recherche français ou étrangers, des laboratoires publics ou privés. 


\title{
Modal coupling in one-dimensional electro- mechanical structured continua
}

\author{
S. Vidoli and F. dell'Isola, Rome, Italy \\ (Received June 23, 1998; revised January 19, 1999)
}

\begin{abstract}
Summary. A passive continuously distributed control of mechanical vibrations is proposed. The piezoelectric actuators are interconnected by a linear electric transmission line. We introduce coupling and internal resonance criteria to determine the optimal choices for electric parameters. These criteria can be found decomposing the differential operator appearing in the linear evolution equations according to a partition of the state vector into mechanical and electrical parts. The results we find allow for the design of an experimental set up.
\end{abstract}

\section{Introduction}

Today many research efforts are devoted to the structural control by electric devices; they are mainly based on the piezoelectric effect (see [1] - [4]).

Several technological and theoretical problems arise:

i) to control vibrations by means of concentrated devices requires high actuator performances (often not yet available) and high control power with risk of electric discharges;

ii) the electric wave-speed is too high compared to the mechanical wave-speed; this means that a real dynamical coupling between electric and mechanical waves (e.g., in a piezoelectric material) is not possible.

Our paper is intended to overcome these two problems:

i) by developing the ideas already discussed in [5] and [6], we introduce a transmission line connecting a series of piezoelectric actuators distributed along the structure. The obtained synergy of actuators seems to improve considerably their control performances: to do the job ten men are better than one alone even if this last is ten times stronger; moreover ten men communicating and helping one another are better than ten men acting alone;

ii) the speed of electric waves can be easily controlled in electric transmission lines by a proper choice of the line inductances and capacitances: the high capacitance of modern PZT actuators allows to push the electric wave-speed into the mechanical range by means of technically feasible line inductances.

The device we propose has a double practical advantage: it requires lower performances to the PZT actuators and it produces an electro-mechanical interaction based on internal resonance phenomena between the modes of the whole electro-mechanical system: this allows for a strong control action and short times to transfer the energy between electrical and mechanical forms.

In Sect. 2 the balance equations for a one-dimensional continua endowed with an affine electro-mechanical structure are derived following the ideas developed in [7]: the mechanical configuration of the beam and the charge distribution in the electric transmission lines are 
chosen as state variables of the system; the mechanical contact actions, the inertial actions and the electric potential drops are introduced as dual quantities of the generalized velocity and deformation fields; balance equations and boundary conditions are finally obtained by means of the principle of virtual power (see [8]).

Section 3 mainly concerns the analytical study of the general linear differential operator of the problem within the partition of the state vector into its mechanical and electrical parts. Criteria for the existence of electro-mechanical coupling are stated, and a representation of the solution by Volterra integral equations allows to understand the coupling mechanism and leads to the proper choice of the electrical parameters such as the line inductance and the line resistance producing internal resonance between electrical and mechanical vibrations. In particular it is proven that mutual excitation between mechanical modes can occur also in the linear case through the electric charge vibrations.

Finally, in Sect. 4 we illustrate the previous results by means of numerical simulations; a purely flexible beam clamped-clamped and electrically insulated is chosen as ad hoc example in a time-evolution problem. In this example the spectral properties of the involved differential operator produce an interesting coupling pattern between electrical and mechanical modes. The results of Sect. 4 allow us to design an experimental set up aiming to prove the technological feasibility of the conceived passive piezoelectric damping.

\section{Balance equations and boundary conditions}

The mechanical behavior of beams is sometimes very complex. It has been proposed to model such a behavior introducing one-dimensional microstructured continua in which a set of directors describes the deformations of the beam sections (see [9]). The most relevant kinematical descriptor of the state of these sections is represented by the attitude which was introduced by Euler and Bernoulli [10]. However, no further conceptual effort is required when modelling sections constrained to undergo affine deformations [7]. In the present paper we introduce a further set of descriptors in order to model electrical transmission lines coupled to the beam by means of a set of distributed piezoelectric actuators. We will adapt to the electromechanical case the ideas developed in [11].

Let $\mathcal{B}$ be the one-dimensional base manifold, $\mathcal{F}$ the two-dimensional point-shape manifold, $\mathcal{Q}$ an $N$-dimensional charges manifold, $\mathcal{P}$ the three-dimensional positions manifold and $V \mathcal{P}$ its translation space. All the manifolds are supposed to be endowed with the euclidean metric.

The configuration is a function:

$\mathcal{B} \times \mathcal{F} \rightarrow \mathcal{P} \times \mathcal{Q} \quad(\varsigma, y) \mapsto(x(\varsigma, y), \mathbf{q}(\varsigma))$,

where $\mathbf{q}(\varsigma)$ represents the electrical charges stored in the $[0, \varsigma]$ segment of the transmission lines, and

$\mathbf{x}(\varsigma, y)=\mathbf{p}(\varsigma)+\mathbf{P}(\varsigma)(y-o)$

is the position of a beam point defined by $\mathbf{p}(\varsigma)=\mathbf{x}(\varsigma, o)$ which physically represents the position of an arbitrary point $o$ on $\mathcal{F}$ chosen as origin, and by $\mathbf{P}(\varsigma)$ which is a linear embedding ${ }^{1}$ of $V \mathcal{F}$ in $V \mathcal{P}$ representing the attitude and the affine stretching of the beam fibers. More precisely, $\forall \varsigma \in \mathcal{B}, \mathbf{x}(\varsigma, \cdot)$ is an affine application of $\mathcal{F}$ in $\mathcal{P}$, invertible on its image $\mathcal{F}_{\varsigma}:=\mathbf{x}(\varsigma, \mathcal{F})$ so that $\mathbf{P}(\varsigma) \in \operatorname{IsoLin}\left(V \mathcal{F}, V \mathcal{F}_{\varsigma}\right)^{2}$.

\footnotetext{
${ }^{1}$ We mean a linear application $V \mathcal{F} \rightarrow V \mathcal{P}$ which is invertible on its image.

2 We use the notation introduced in [12]: LIN is the set of linear applications, IsoLIN the set of linear invertible applications, SYM and SKW respectively the set of symmetric and skewsymmetric linear applications. We will label by SYM $\mathbf{A}$ and SKW $\mathbf{A}$ the symmetric and skewsymmetric parts of $\mathbf{A}$.
} 
A motion is a one-parameter family of configurations:

$(\varsigma, y, \tau) \mapsto[\mathbf{x}(\varsigma, y, \tau), \mathbf{q}(\varsigma, \tau)]=[\mathbf{p}(\varsigma, \tau)+\mathbf{P}(\varsigma, \tau)(y-o), \mathbf{q}(\varsigma, \tau)]$

For any pair of time instants $(t, \tau)$ it is useful to consider the following equations:

$\mathbf{x}(\tau)-\mathbf{p}(\tau)=\mathbf{P}(\tau) \mathbf{P}(t)^{-1}[\mathbf{x}(t)-\mathbf{p}(t)]$

describing the evolution of the fiber vectors. The time derivative of Eq. (2.4) gives us the velocity of the point:

$\dot{\mathbf{x}}=\dot{\mathbf{p}}+\dot{\mathbf{P}} \mathbf{P}^{-1}(\mathbf{x}-\mathbf{p})=\mathbf{w}+\mathbf{W}(\mathbf{x}-\mathbf{p})$,

so we are led to define the generalized velocity field as

$(\mathbf{w}, \mathbf{W}, \omega):=\left(\dot{\mathbf{p}}, \dot{\mathbf{P}} \mathbf{P}^{-1}, \dot{\mathbf{q}}\right)$.

We note that $\omega=\dot{\mathrm{q}}$ is a set of electric current fields. Also we remark that the tensor $\mathbf{W}:=\dot{\mathbf{P}} \mathbf{P}^{-1} \in \operatorname{IsoLIN}\left(V \mathcal{F}_{\varsigma, t}, V \mathcal{F}_{\kappa, \tau}\right)$ maps the plane copy of the beam fiber at time $t$ in the copy at time $\tau$; we can extend its action to the whole translation by means of the condition:

$\mathbf{W} \mathbf{n}(\varsigma, \tau):=-\mathbf{W}^{T} \mathbf{n}(\varsigma, \tau)$,

being $\mathbf{n}(\varsigma, \tau)$ the unit normal to $V \mathcal{F}_{\varsigma, \tau}$.

We choose as descriptor of the time-rate of the generalized deformation the following field:

$(\mathbf{d}, \mathbf{D}, \boldsymbol{\Delta}, \boldsymbol{\delta}):=\left(\mathrm{W}^{\prime}-(\mathrm{SkW} \mathbf{W}) \mathbf{p}^{\prime}, \mathbf{W}^{\prime}, \operatorname{SYMW}, \boldsymbol{\omega}^{\prime}\right)$,

which vanishes for every velocity field mechanically rigid and with spatially constant current fields. Here the prime means the derivative with respect to $\varsigma$.

We call internal actions its dual quantity $(\mathbf{s}, \mathbf{S}, \boldsymbol{\Sigma}, \boldsymbol{\sigma})$ in the expression of the internal power $P_{i}$ :

$P_{i}=\int_{\mathcal{B}}(\mathbf{s} \cdot \mathbf{d}+\mathbf{S} \cdot \mathbf{D}+\mathbf{\Sigma} \cdot \mathbf{\Delta}+\boldsymbol{\sigma} \cdot \boldsymbol{\delta}) d \varsigma$,

where "." denotes the inner product in the set of vectors or tensors (see, e.g., [12]). While s and $\mathbf{S}$ represent the standard contact force and couple, the dual quantities $\boldsymbol{\Sigma}$ and $\boldsymbol{\sigma}$ represent the contact double force exerted on the beam fiber and the set of local electric potential drops, respectively.

The balance equations can be evaluated assuming that for any generalized velocity field the power $P_{e}$ expended by the external actions, $(\mathbf{b}, \mathbf{B}, \boldsymbol{\beta})$, equals the internal power:

$P_{i}=\int_{\mathcal{B}}(\mathbf{s} \cdot \mathbf{d}+\mathbf{S} \cdot \mathbf{D}+\boldsymbol{\Sigma} \cdot \boldsymbol{\Delta}+\boldsymbol{\sigma} \cdot \boldsymbol{\delta}) d \varsigma=\int_{\mathcal{B}}(\mathbf{b} \cdot \mathbf{w}+\mathbf{B} \cdot \mathbf{W}+\boldsymbol{\beta} \cdot \boldsymbol{\omega}) d \varsigma=P_{e}$

Substituting Eq. (2.8) in Eq. (2.10), integrating by parts and recalling that ${ }^{3} \mathbf{a} \cdot \mathbf{A b}=\mathbf{A} \cdot \mathbf{a} \wedge \mathbf{b}$ for every $\mathbf{A} \in \mathrm{S}_{\mathrm{K} w}$, we get:

$\int_{\mathcal{B}}\left[\left(\mathbf{s}^{\prime}+\mathbf{b}\right) \cdot \mathbf{w}+\left(\mathbf{S}^{\prime}+\mathbf{\Sigma}+\mathbf{p}^{\prime} \wedge \mathbf{s}+\mathbf{B}\right) \cdot \mathbf{W}+\left(\boldsymbol{\sigma}^{\prime}+\boldsymbol{\beta}\right) \cdot \boldsymbol{\omega}\right] d \varsigma=[\mathbf{s} \cdot \mathbf{w}+\mathbf{S} \cdot \mathbf{W}+\boldsymbol{\sigma} \cdot \boldsymbol{\omega}]_{\partial \mathcal{B}}$,

that must vanish for every regular velocity field $(\mathbf{w}, \mathbf{W}, \boldsymbol{\omega})$. As a consequence for every $\varsigma \in \mathcal{B}$ :

$\mathbf{s}^{\prime}+\mathbf{b}=\mathbf{0}, \quad(\operatorname{skw} \mathbf{S})^{\prime}+\mathbf{p}^{\prime} \wedge \mathbf{s}+\mathrm{skW} \mathbf{B}=\mathbf{0}$,

$(\operatorname{SYM} \mathbf{S})^{\prime}+\mathbf{\Sigma}+\operatorname{SYM} \mathbf{B}=\mathbf{0}, \quad \boldsymbol{\sigma}^{\prime}+\boldsymbol{\beta}=\mathbf{0}$,

${ }^{3} \mathbf{a} \wedge \mathbf{b}=\mathbf{a} \otimes \mathbf{b}-\mathbf{b} \otimes \mathbf{a}$ 
and for $\varsigma \in \partial \mathcal{B}$ :

$[\mathbf{s} \cdot \mathbf{W}+\mathbf{S} \cdot \mathbf{W}+\boldsymbol{\sigma} \cdot \boldsymbol{\omega}]_{5}=0$.

Equations (2.11.1-3) and (2.12) evaluated for $\boldsymbol{\omega}=0$ are the standard balance equations of a one-dimensional continuum endowed with affine structure. Equations (2.11.4) and (2.12) evaluated for $(\mathbf{w}, \mathbf{W})=(\mathbf{0}, 0)$ give a formulation of Tellegen theorem (see [13]). The problem will be complete when the constitutive relations for $(\mathbf{s}, \mathbf{S}, \mathbf{\Sigma}, \boldsymbol{\sigma})$ and $(\mathbf{b}, \mathbf{B}, \boldsymbol{\beta})$ are assigned.

\section{Electro-mechanical coupling criteria}

In the following we will limit our attention to the linearized model and rearrange in vectors (labeled by a tilde) the strict components of the infinitesimal displacement $\tilde{\mathbf{u}}$, of the deformation field $\tilde{\mathbf{d}}$, and of the internal and external actions ( $\tilde{\mathbf{s}}$ and $\tilde{\mathbf{b}})$. The compatibility Eqs. $(2.8)$ can be linearized getting

$\tilde{\mathrm{d}}=\mathbf{G}(\tilde{\dot{\mathbf{u}}})$,

where $\mathrm{G}$ is the linear differential operator which determines the time-rate generalized deformation field. The linear constitutive equations can be written in the form:

$\tilde{\mathbf{s}}=\mathbf{K G}(\tilde{\mathbf{u}}), \quad \tilde{\mathbf{b}}=-\mathbf{M} \tilde{\tilde{\mathbf{u}}}$,

where $\mathbf{K}$ and $\mathbf{M}$ are the symmetric positive definite stiffness and inertia matrices; moreover we will assume that $\mathbf{M}$ is diagonal. Recalling Eq. (2.10) we get

$\int_{\mathcal{B}}[\mathbf{K G}(\tilde{\mathbf{u}}) \cdot \mathbf{G}(\tilde{\mathbf{w}})] d \varsigma=\int_{\mathcal{B}}\left[\mathbf{G}^{A} \mathbf{K G}(\tilde{\mathbf{u}}) \cdot \tilde{\mathbf{w}}\right] d \varsigma=-\int_{\mathcal{B}}[\mathbf{M} \tilde{\tilde{\mathbf{u}}} \cdot \tilde{\mathbf{w}}] d \varsigma, \quad \forall \tilde{\mathbf{w}}$,

so that the linearized balance equations in terms of infinitesimal displacement fields become

$\mathbf{L}(\tilde{\mathbf{u}})+\tilde{\mathbf{u}}=0$,

where $\mathbf{L}:=\mathbf{M}^{-1} \mathbf{G}^{A} \mathbf{K} \mathbf{G}$.

\subsection{Modal analysis of partitioned operator $L$}

We study Eq. (3.4) partitioning $\tilde{\mathbf{u}}$ in its mechanical and electrical components and we label respectively by $m$ and $e$ indices quantities of mechanical and electrical nature. For sake of simplicity we consider $\tilde{\mathbf{u}}$ as a two-dimensional vector.

Let $\mathcal{A}$ be the Hilbert space:

$\mathcal{A}:=\mathcal{A}_{m} \times \mathcal{A}_{e}$

where $\mathcal{A}_{m}$ and $\mathcal{A}_{e}$ are subspaces of $\mathbf{L}^{2}(\mathcal{B})$, the space of $\mathbb{R}$-valued square-integrable functions defined on $\mathcal{B}$ verifying suitable homogeneous boundary conditions, endowed with the usual inner product $\langle\square, \diamond\rangle:=\int_{\mathcal{B}}(\square \diamond) d \varsigma$; let $\mathbf{u}, \ddot{\mathbf{u}} \in \mathcal{A}$ and let $\mathbf{L}$ be a self-adjoint positive definite differential operator on $\mathcal{A}$. We consider the following evolutionary problem:

$\mathbf{L}(\mathbf{u})+\ddot{\mathbf{u}}=0, \quad \mathbf{u}(0)=\mathbf{u}_{0}, \quad \dot{\mathbf{u}}(0)=\dot{\mathbf{u}}_{0}$. 
The partition (3.5) induces the following decompositions of the state vector $\mathbf{u}$ and of Eq. (3.6.1):

$\mathrm{u}=\{v, \gamma\}, \quad v \in \mathcal{A}_{m}, \quad \gamma \in \mathcal{A}_{e}$,
$L_{m m}(v)+L_{m e}(\gamma)+\ddot{v}=0, \quad L_{e m}(v)+L_{e e}(\gamma)+\ddot{\gamma}=0$,

where the operators $L_{m m}$ and $L_{e e}$ are self-adjoint and $L_{m e}$ is the adjoint of $L_{e m}$ because of the selfadjointness of $\mathbf{L}$.

Equations (3.8) lead us to regard $v$ as the $m$-response to $m$ inertial actions but also to the $e$-action of $L_{m e}(\gamma)$ and viceversa for $\gamma$. Let us determine in a more expressive form this dependence; as a consequence of the spectral representations theorem of self-adjoint oparators (see [14]), for every $a \in \mathcal{A}_{m}, b \in \mathcal{A}_{e}$ and $r \in \mathbb{R}$ we have

$L_{m m}^{r}(a)=\int_{S_{m}} \lambda^{r}\left\langle a, m_{\lambda}\right\rangle m_{\lambda} d \mu_{\lambda}$,

$b=L_{e e}^{0}(b)=\int_{S_{e}}\left\langle b, e_{\nu}\right\rangle e_{\nu} d \mu_{\nu}$,

where $\left(s_{m}, d \mu_{\lambda}\right)$ and $\left(S_{e}, d \mu_{\nu}\right)$ are the spectral measure spaces respectively for $L_{m m}$ and $L_{e e}$, while $m_{\lambda}$ and $e_{\nu}$ are the corresponding sets of spectral vectors.

Let us define the functions

$v_{\lambda}=\left\langle v, m_{\lambda}\right\rangle$,

$\gamma_{\nu}=\left\langle\gamma, e_{\nu}\right\rangle$,

dependent on the time variable only, and the scalar numbers

$A_{\lambda \nu}=\left\langle m_{\lambda}, L_{m e}\left(e_{\nu}\right)\right\rangle$,

$A_{\nu \lambda}=\left\langle e_{\nu}, L_{e m}\left(m_{\lambda}\right)\right\rangle$,

with $A_{\lambda \nu}=A_{\nu \lambda}$ due to $L_{m e}=L_{e m}^{A}$.

Equations (3.8) can be written as

$\int_{S_{m}}\left(\lambda v_{\lambda}+\ddot{u}_{\lambda}+\int_{S_{e}} A_{\lambda \nu} \gamma_{\nu} d \mu_{\nu}\right) m_{\lambda} d \mu_{\lambda}=0, \quad \int_{S_{e}}\left(\nu \gamma_{\nu}+\ddot{\gamma}_{\nu}+\int_{S_{m}} A_{\nu \lambda} v_{\lambda} d \mu_{\lambda}\right) e_{\nu} d \mu_{\nu}=0$,

which - as $m_{\lambda}$ and $e_{\nu}$ are bases of $\mathcal{A}_{m}$ and $\mathcal{A}_{e}$ respectively - are equivalent to

$\lambda v_{\lambda}+\ddot{u}_{\lambda}+\int_{S_{e}} A_{\lambda \nu} \gamma_{\nu} d \mu_{\nu}=0, \quad \forall \lambda \in S_{m}$,

$\nu \gamma_{\nu}+\ddot{\gamma}_{\nu}+\int_{S_{m}} A_{\nu \lambda} v_{\lambda} d \mu_{\lambda}=0, \quad \forall \nu \in S_{e}$

Equation (3.16) clearly shows that the influence on the mode $m_{\lambda}$ exerted by the mode $e_{\nu}$ is measured by

$A_{\lambda \nu}=\int_{\mathcal{B}} m_{\lambda} L_{m e}\left(e_{\nu}\right) d \varsigma$,

that we can regard as a modal $e \rightarrow m$ coupling matrix. In an absolutely similar way $A_{\nu \lambda}$ represents the modal $m \rightarrow e$ coupling matrix; it is now easy to formulate the following:

Criterion of electro-mechanical coupling

A necessary condition for the presence of electro-mechanical energy exchange between $e_{\nu}$ and $m_{\lambda}$ modes is

$\int_{\mathcal{B}} L_{m e}\left(e_{\nu}\right) m_{\lambda} d \varsigma=\int_{\mathcal{B}} L_{e m}\left(m_{\lambda}\right) e_{\nu} d \varsigma \neq 0$. 


\subsection{Coupling representation by integral Volterra kernels}

In order to test the coupling properties of Eq. (3.6), we search the solution corresponding to purely mechanical initial data:

$v_{\lambda}=v_{\lambda 0}, \quad \dot{v}_{\lambda}=\dot{v}_{\lambda 0} \quad \forall \lambda \in S_{m}$,
$\gamma_{\nu}=0, \quad \dot{\gamma}_{\nu}=0 \quad \forall \nu \in S_{e}$.

We get

$v_{\lambda}(t)=\frac{v_{\lambda 0}}{\cos \phi_{\lambda}} \cos \left(\varpi_{\lambda} t+\phi_{\lambda}\right)+\int_{0}^{t} \frac{1}{\varpi_{\lambda}} \sin \varpi_{\lambda}(t-\check{t}) \int_{S_{e}} A_{\lambda \nu} \gamma_{\nu}(\check{t}) d \mu_{\nu} d \check{t}$

$\gamma_{\nu}(t)=\int_{0}^{t} \frac{1}{\varpi_{\lambda}} \sin \varpi_{\nu}(t-\check{t}) \int_{S_{m}} A_{\nu k} v_{\kappa}(\check{t}) d \mu_{\kappa} d \check{t}$

where $\varpi_{\lambda}:=\sqrt{\lambda}, \varpi_{\nu}:=\sqrt{\nu}$, and $\phi_{\lambda}:=\arctan \left(\dot{v}_{\lambda 0} /\left(\varpi_{\lambda} v_{\lambda 0}\right)\right)$.

Introducing Eq. (3.21.2) to (3.21.1):

$$
\begin{aligned}
v_{\lambda}(t)= & \frac{v_{\lambda 0}}{\cos \phi_{\lambda}} \cos \left(\varpi_{\lambda} t+\phi_{\lambda}\right) \\
& +\int_{0}^{t} \frac{\sin \varpi_{\lambda}(t-\check{t})}{\varpi_{\lambda}} \int_{S_{e}} A_{\lambda \nu} \int_{0}^{\bar{t}} \frac{\sin \varpi_{\nu}(\check{t}-\tau)}{\varpi_{\nu}} \int_{S_{m}} A_{\nu x} v_{x}(\tau) d \mu_{x} d \tau d \mu_{\nu} d \check{t}
\end{aligned}
$$

for every $\lambda \in S_{m}$.

Applying Fubini's theorem to the second member integral and changing the integration order where it is possible, we get

$\int_{0}^{t} \int_{S_{m}}\left[\int_{S_{\mathrm{e}}} \frac{A_{\lambda \nu} A_{\nu k}}{\varpi_{\lambda} \varpi_{\nu}} \int_{\tau}^{t} \sin \varpi_{\lambda}(t-\check{t}) \sin \varpi_{\nu}(\check{t}-\tau) d \check{t} d \mu_{\nu}\right] v_{k}(\tau) d \mu_{k} d \tau$.

The term in the square brackets can be evaluated to get

$$
\begin{aligned}
\mathbb{K}_{\lambda_{1} x}(t, \tau) & =\int_{S_{e}} \frac{A_{\lambda \nu} A_{\nu x}}{\varpi_{\lambda} \varpi_{\nu}} \int_{\tau}^{t} \sin \varpi_{\lambda}(t-\check{t}) \sin \varpi_{\nu}(\check{t}-\tau) d \check{t} d \mu_{\nu} \\
& =\int_{S_{e}} \frac{A_{\lambda \nu} A_{\nu x}}{\varpi_{\lambda} \varpi_{\nu}} \frac{\varpi_{\lambda} \sin \varpi_{\nu}(t-\tau)-\varpi_{\nu} \sin \varpi_{\lambda}(t-\tau)}{\varpi_{\lambda}{ }^{2}-\varpi_{\nu}{ }^{2}} d \mu_{\nu},
\end{aligned}
$$

so we are led to recognize (3.22) as a system of Volterra integral equations of the second kind:

$v_{\lambda}(t)=f_{\lambda}(t)+\int_{0}^{t} \int_{S_{m}} \mathbb{k}_{\lambda_{,}, x}(t, \tau) v_{\varkappa}(\tau) d \mu_{x} d \tau$

in which $f_{\lambda}(t)$ represents the standard cosinusoidal evolutions of the mechanical modes, and the integral is the representation of the effects of the back and forth exchange of energy with the electrical system ${ }^{4}$.

4 The solution of the system of equations (3.25) can be easily derived by the following constructive iterative scheme (see [15]): $v_{\lambda}{ }^{(0)}(t)=f_{\lambda}(t), v_{\lambda}{ }^{(n)}(t)=f_{\lambda}(t)+\int_{0}^{t} \mathbb{k}_{\lambda, x}(t, \tau) v_{x}{ }^{(n-1)}(\tau) d \tau$, if $f(t)$ is continuous in $[0, T]$ and the kernel $\mathbb{k}(t, \tau)$ is continuous for $t \in[0, \stackrel{0}{T}]$ and $\tau \in[0, t]$ (which is verified trivially for Eq. (3.24)). 
We remark that Eq. (3.25) shows that the evolution of the mechanical mode $\lambda$ (eigenfunction of the operator $L_{m m}$ ) can be influenced by the evolution of all mechanical modes. Because of this property mechanical modal excitation can occur via electro-mechanical energy exchange.

The structure of the kernel $\mathrm{k}_{\lambda, x}$ defined in Eq. (3.24) plays a crucial role to determine the kind of coupling involved: two mechanical modes $\lambda$ and $x$ can influence one another if and only if the number $A_{\lambda \nu} A_{\nu, x}$ is non-vanishing (i.e., they have a non-vanishing coupling with almost one electrical mode $\nu$ ).

Moreover, Eq. (3.24) shows that for $\varpi_{\nu} \rightarrow \varpi_{\lambda}$ the Volterra kernel $\mathbb{k}_{\lambda, \mathrm{k}}$ becomes resonant; this fact is easily checked by recalling that

$\lim _{\varpi_{\nu} \rightarrow \varpi_{\lambda}} \frac{\varpi_{\lambda} \sin \varpi_{\nu}(t-\tau)-\varpi_{\nu} \sin \varpi_{\lambda}(t-\tau)}{\varpi_{\lambda}^{2}-\varpi_{\nu}^{2}}=\frac{1}{2}\left[\frac{\sin \varpi_{\lambda}(t-\tau)}{\varpi_{\lambda}}+(\tau-t) \cos \varpi_{\lambda}(t-\tau)\right]$.

This fact leads us to expect a strong coupling in the time-evolutions of $\lambda$ (mechanical) and $\nu$ (electrical) modes when the corresponding pulsations $w$ are close. Finally, in order to have a dimensionless number measuring the coupling, as consequence of the positive definiteness of $\mathbf{L}$, we introduce the coupling coefficients as the numbers:

$c_{\lambda \nu}:=\left|\frac{2 A_{\lambda \nu}}{\varpi_{\lambda}+\varpi_{\nu}}\right|<1$.

Equation (3.27) follows from $\langle\mathbf{L} \mathbf{u}, \mathbf{u}\rangle>0$ for $\mathbf{u}=\left\{m_{\lambda}, e_{\nu}\right\}$ and for $\mathbf{u}=\left\{m_{\lambda},-e_{\nu}\right\}$. The subsequent numerical simulations prove that the closer the number $c_{\lambda \nu}$ is to 1 , the bigger is the coupling between $\lambda$ and $\nu$ modes.

\section{Applications}

The model described in the previous sections can be used to study the behavior of several electro-mechanical systems. In the papers [5] and [6] truss modular beams controlled by distributed PZT actuators were considered. In the present paper we address to the dynamical control of a continuous beam.

\subsection{System description}

In order to prove the feasibility and the efficiency of the distributed PZT control, we consider an experimental set up $^{5}$ made of an aluminum beam coupled with a set of piezoelectric patch actuators spatially distributed on its upper surface (see Fig. 1).

The Aluminum beam, we consider in the numerical simulations, is $60 \mathrm{~cm}$ long, $2.3 \mathrm{~cm}$ large and $3 \mathrm{~mm}$ thick. We will regard it as a Bernoulli-Navier purely flexible beam.

The actuators are connected in series as shown by Fig. 2 by means of impedances, in such a way that a transmission line is formed. The line capacitance is constituted by the actuators only. The beam is clamped-clamped, while the transmission line connecting the actuators is electrically insulated.

\footnotetext{
5 which is being realized in a laboratory of the "Università dell" Aquila".
} 


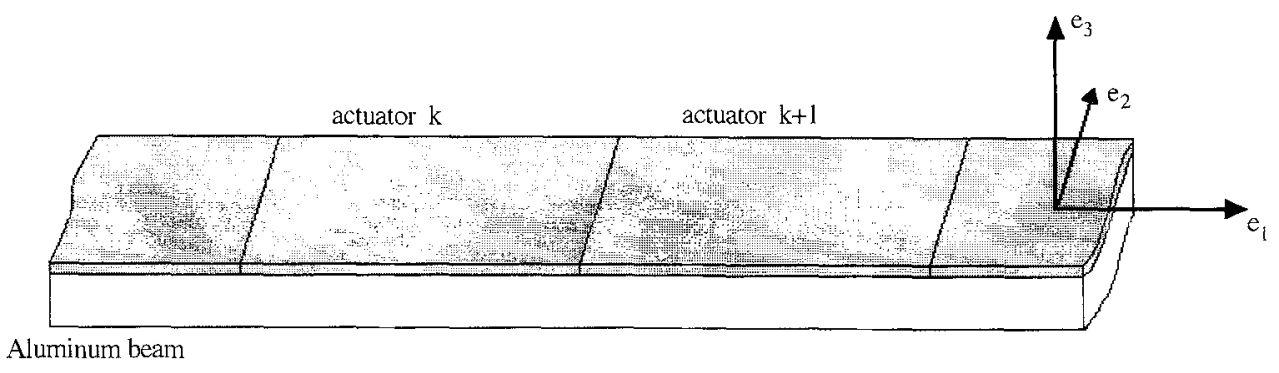

Fig. 1. Beam and actuators setting

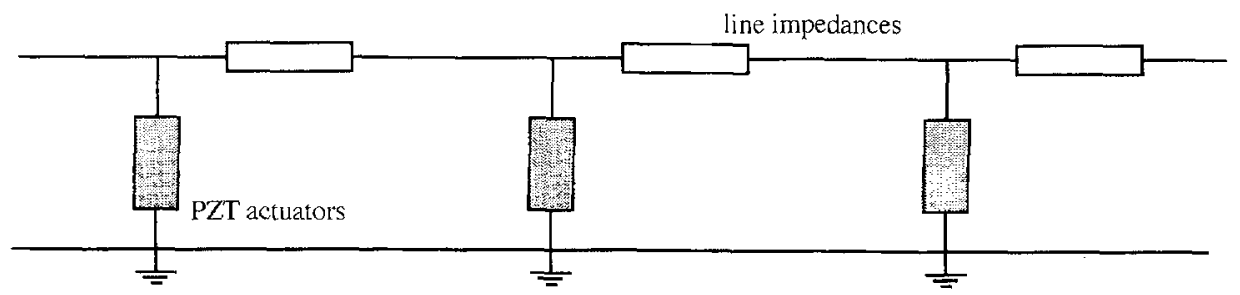

Fig. 2. Electric line scheme (gray boxes for PZT actuators)

In our simulations we consider the physical properties of commercial actuators (namely ACX QuickPack actuators) in bonded configuration: this means that the actuators are supposed to supply non-vanishing forces and vanishing couples when subjected to voltages.

\subsection{Identification of continuum constitutive parameters}

Let us choose as vector of kinematical descriptors

$\tilde{\mathbf{u}}=\{V, \Gamma\}$,

being $V$ the transverse displacement of the beam section and $\Gamma$ the charge stored in the segment $[0, \varsigma]$ of the actuators transmission line. Limiting our analysis to the case of a purely flexible beam the strict components of the deformation time-rate are given by

$\tilde{\mathrm{d}}=\left\{D_{13}, \delta\right\}=\left\{\dot{V}^{\prime \prime}, \dot{\Gamma}^{\prime}\right\} \Longrightarrow \mathbf{G}=\left(\begin{array}{cc}\square^{\prime \prime} & 0 \\ 0 & \square^{\prime}\end{array}\right)$,

where ${ }^{6} D_{13}=\dot{V}^{\prime \prime}$ is the field of beam curvature time-rate and $\delta=\dot{\Gamma}^{\prime}$ is the field of currents in the PZT actuators. Their dual actions will be

$\check{\mathrm{s}}=\left\{S_{13}, \sigma\right\}$,

physically representing the bending moment field $S_{13}$ and the potential drop field $\sigma$ at the extreme points of the PZT actuators.

Due to their positioning over the beam, these actuators supply a bending moment when subjected to voltage fields and vice versa: this coupling effect can be mathematically represent-

6 Here the tensor $\mathbf{D}$ is represented in the basis shown in Fig. 1. 
ed in a coupled constitutive relation between the internal actions $\tilde{\mathrm{s}}$ and the deformation fields $\mathbf{G}(\tilde{\mathbf{u}})$ :

$\tilde{\mathbf{s}}=\mathbf{K G}(\tilde{\mathbf{u}}), \quad \mathbf{K}=\left(\begin{array}{cc}K_{m m} & K_{m e} \\ K_{e m} & K_{e e}\end{array}\right)$.

We will assume that

$K_{m m}=E I+k_{m m} \bar{y}^{2}, \quad K_{m e}=k_{m e} \bar{y}=k_{e m} \bar{y}=K_{e m}, \quad K_{e e}=k_{e e}$,

being $E$ the Young modulus of the aluminum beam, $I$ the moment of inertia of its section and $\bar{y}=1.65 \mathrm{~mm}$ the distance of the patch actuators from the center axis of the beam. The quantities $k_{m m}, k_{m e}=k_{e m}$ and $k_{e e}$ are respectively the axial stiffness, the electro-mechanical coupling constants and the admittance for unit length of the actuators as read from the ACX catalogue (i.e., $k_{m m}=2.10^{5} \mathrm{~N}, k_{m e}=k_{e m}=0.410^{5} \mathrm{~V}, k_{e e}=2.210^{5} \mathrm{~m} /$ Farad).

Moreover, recalling that the electrical "inertial" actions physically represent the lines potential drop, we can write Eq. (3.2.2) as

$\tilde{\mathbf{b}}=-\{\varrho \ddot{V}, h \ddot{\Gamma}\} \Longrightarrow \mathbf{M}=\left(\begin{array}{ll}\varrho & 0 \\ 0 & h\end{array}\right)$

where $\varrho$ is the linear mass density of the beam and $h$ is the line inductance. With the stated constitutive relations, Eq. (3.4) becomes

$K_{m m} V^{I V}+K_{m e} \Gamma^{\prime \prime \prime}+\varrho \ddot{V}=0, \quad-K_{e m} V^{\prime \prime \prime}-K_{e e} \Gamma^{\prime \prime}+h \ddot{\Gamma}=0$.

In order to apply the results of Sect. 3 we compute a dimensionless form of Eqs. (4.7) introducing

$v=\frac{V}{l_{b}}, \quad \gamma=\frac{\Gamma}{\bar{q}}$,

where $l_{b}$ is the total length of the beam considered and $\bar{q}:=\varrho l_{b}{ }^{2} / h$ is the reference charge leaving unchanged the symmetry of the spatial differential operator involved. Finally for the time derivatives the pulsation

$\bar{\omega}=(4.73)^{2} \sqrt{\frac{K_{m m}}{\varrho l_{b}^{4}}}$,

of the first mode of the clamped-clamped uncoupled beam is used.

In dimensionless coordinates the operator $\mathbf{L}$ introduced by (3.4) becomes

$\mathbf{L}=\left(\begin{array}{cc}\frac{K_{m m}}{\bar{\omega}^{2} l_{b}^{4}} \square^{\mathrm{IV}} & \frac{K_{m e}}{\bar{\omega}^{2} l_{b}{ }^{2} h} \square^{\mathrm{III}} \\ -\frac{K_{e m}}{\bar{\omega}^{2} l_{b}{ }^{2} h} \square^{\mathrm{III}} & -\frac{K_{e e} \varrho}{\bar{\omega}^{2} h^{2}} \square^{\mathrm{II}}\end{array}\right)$.

\subsection{Numerical simulations}

We consider in this section the spectra $S_{m}$ and $S_{e}$ of the operators $L_{m m}$ and $L_{e e}$ associated with Eq. (4.10) and the boundary conditions specified in Sect. 4.1. These spectra are discrete sets $\{. . \lambda .\},.\{. . \nu .$.$\} of real positive numbers which we can order using natural numbers.$

Once remarked that the electric spectrum is a function of the line inductance, we estimate the critical line inductances $h_{\lambda \nu}^{*}$, maximizing the electro-mechanical mode couplings, assuming vanishing $K_{m e}$ and solving the equation $\varpi_{\nu}(h)=\varpi_{\lambda}$ as is suggested by Eq. (3.26). In this way 
we can construct a matrix of critical values for line inductance such that the electrical pulsation is closest to the mechanical one; for the examined system we get

$h^{*}=\left(\begin{array}{cccccc}\ldots & 4.19 & \ldots & 8.38 & \ldots & 12.57 \\ 0.76 & \ldots & 2.28 & \ldots & 3.80 & \ldots \\ \ldots & 0.78 & \ldots & 1.55 & \ldots & 2.36 \\ 0.23 & \ldots & 0.70 & \ldots & 1.17 & \ldots \\ \ldots & 0.31 & \ldots & 0.63 & \ldots & 0.94 \\ 0.10 & \ldots & 0.31 & \ldots & 0.51 & \ldots\end{array}\right) \mathrm{H} / \mathrm{m}$

whose entry $h_{i j}$ means the inductance maximizing coupling of $i$-mechanical with $j$-electrical modes, and some dots are used when the necessary coupling condition (3.18) is not verified.

In Fig. 3 we plot the spectrum of $\mathrm{L}$ as a function of the line inductance $h$. The obtained set of curves shows a veering phenomenon in the neighborhood of every critical line inductance. The veering regions are characterized by circles. The intersections of spectral curves occur only when $c_{\lambda \nu}=0$, i.e., when the necessary coupling condition is not verified. In Fig. 4 the matrix of coupling coefficients $c_{\lambda \nu}$ is shown; black means 1 , white 0 , and the intensity of gray is proportional to the estimated value.

Several numerical simulations are performed to obtain time evolutions of the first four mechanical and four electrical modes corresponding to the initial data:

$v_{1}=1, \quad v_{i}=0, \quad \gamma_{j}=0, \quad i=2, \ldots 4, \quad j=1, \ldots 4$

of the modal coefficients.

In Fig. $5 h=h_{12}^{*}$, and the line is assumed to be non-dissipative. We observe that:

i) only odd mechanical and even electrical modes are excited, as suggested by the coupling matrix shown in Fig. 4;

ii) the amplitudes of vibrations decrease with increasing modes;

iii) the mechanical vibration of the third mode is excited via the excitations of the (even) electrical ones.

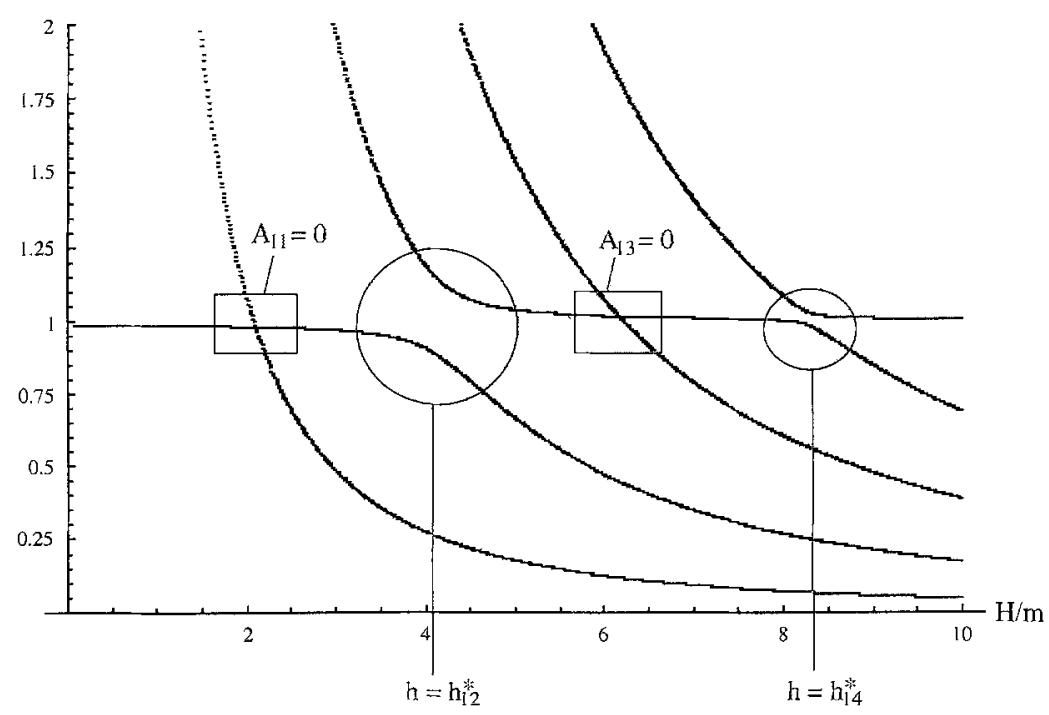

Fig. 3. Veering of eigenfrequencies (normalized with respect to $\bar{\omega}$ ) at the critical line inductances 


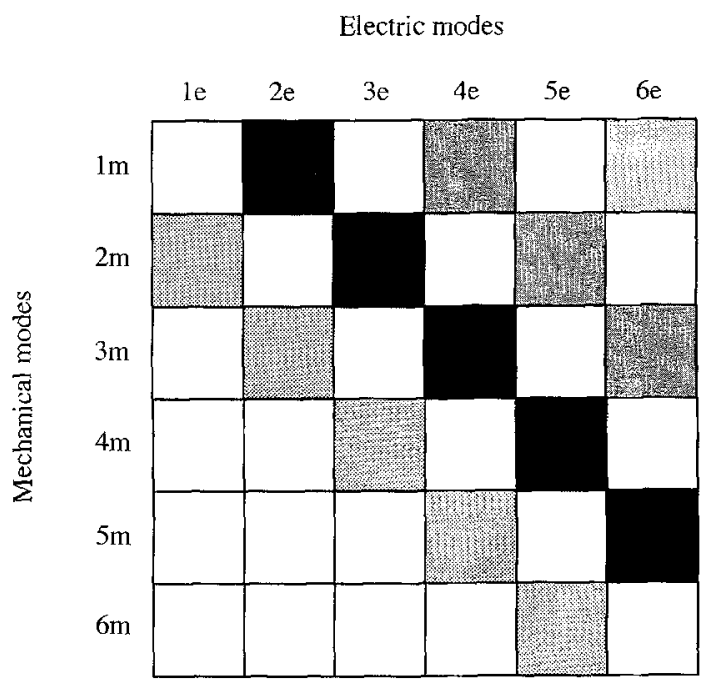

Fig. 4. Coupling coefficients matrix
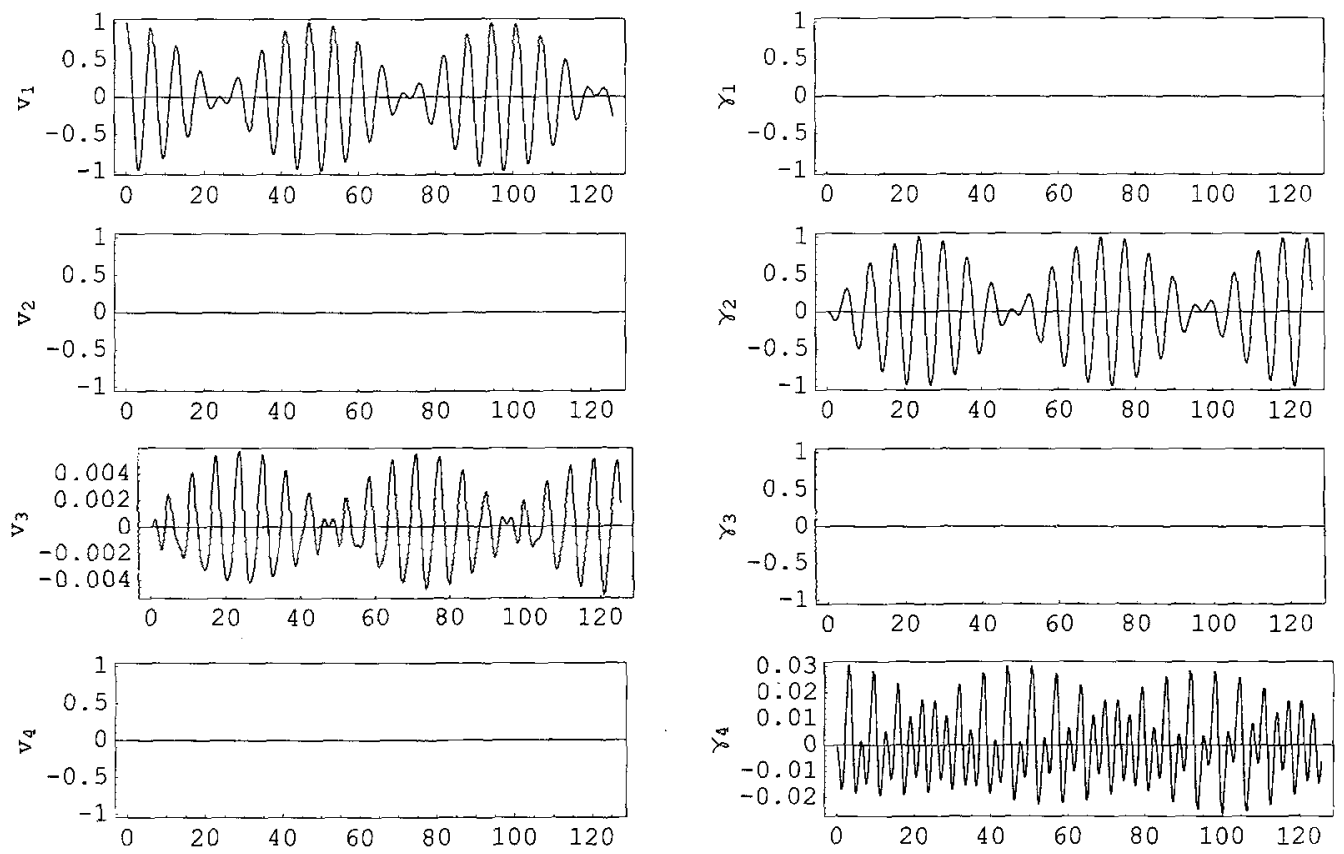

Fig. 5. Time evolution simulation ( $h=h_{12}^{*}$, non-dissipative line)

In Fig. $6, h=h_{12}^{*}$, but the transmission line is assumed to be dissipative, its resistance being $101 \Omega / \mathrm{m}$ (for more details about the optimal choice of the line resistance we refer to [5] and [6]). The vibration of the first mechanical mode is damped as follows: the even electrical modes ( 2 and 4 are shown in the figure) are excited; as a consequence the odd mechanical modes ( 1 and 3 in the figure) are also excited; the line resistance dissipates the electrical energy which cannot be pulled back in the mechanical form. 

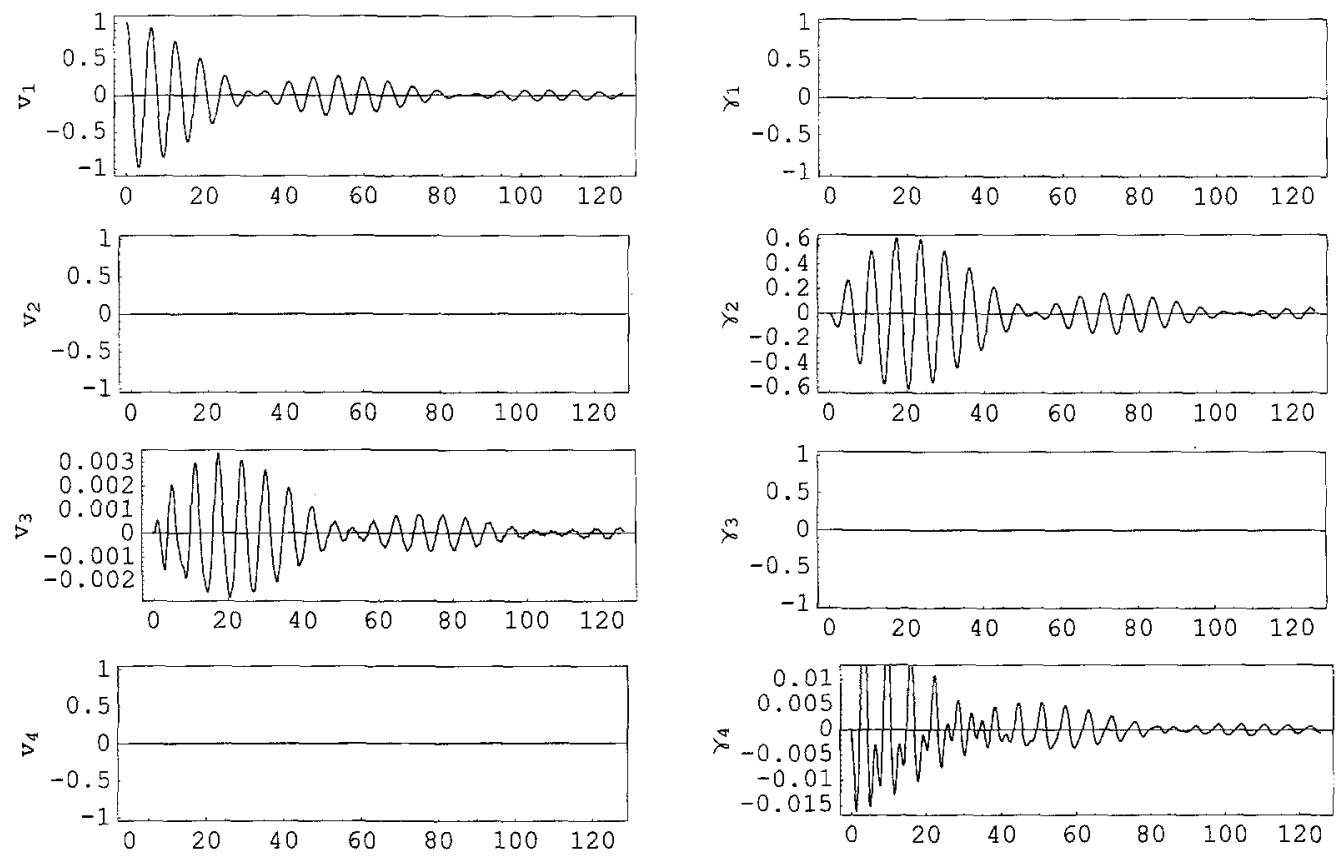

Fig. 6. Time evolution simulation ( $h=h_{12}^{*}$, dissipative line)
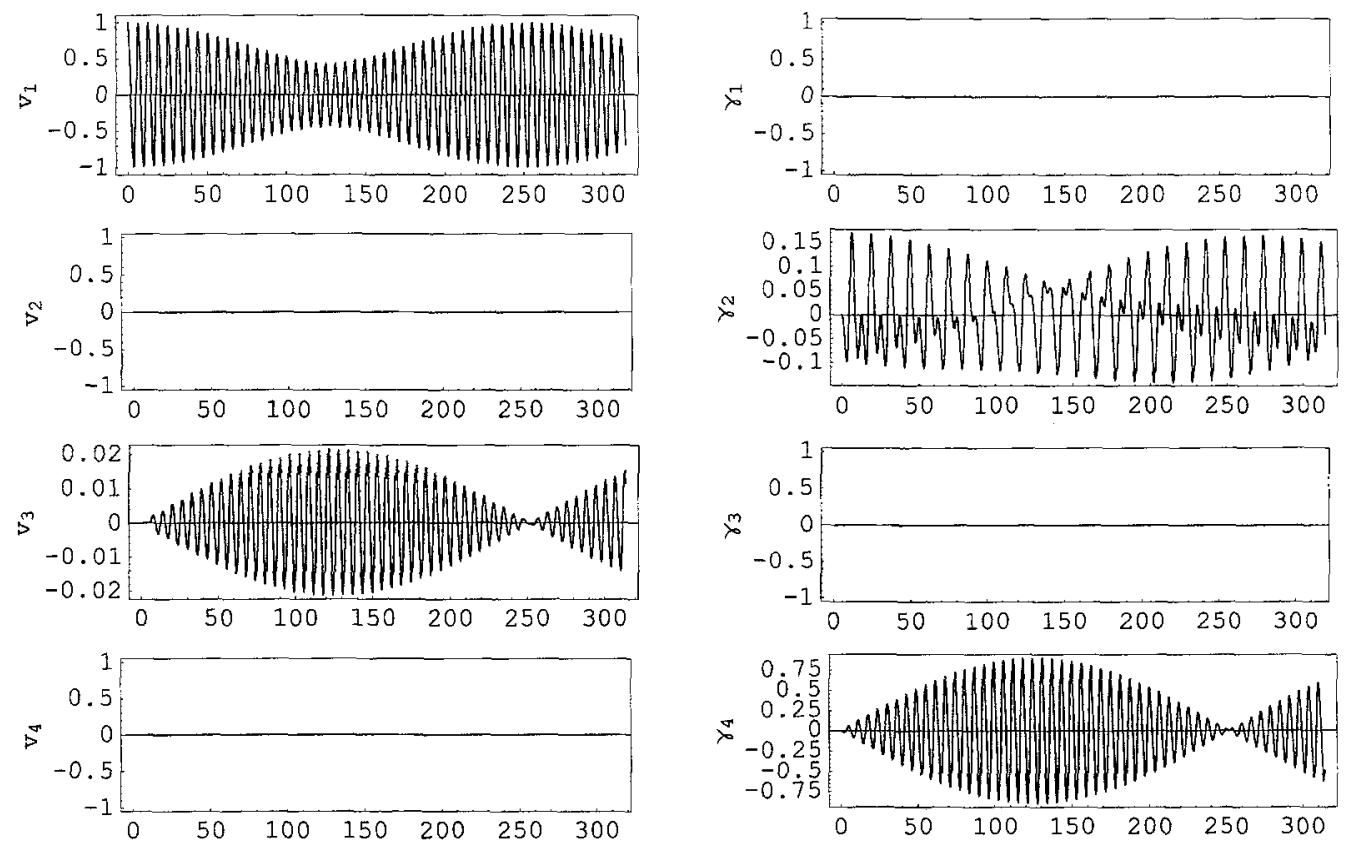

Fig. 7. Time evolution simulation ( $h=h_{14}^{*}$, non-dissipative line) 

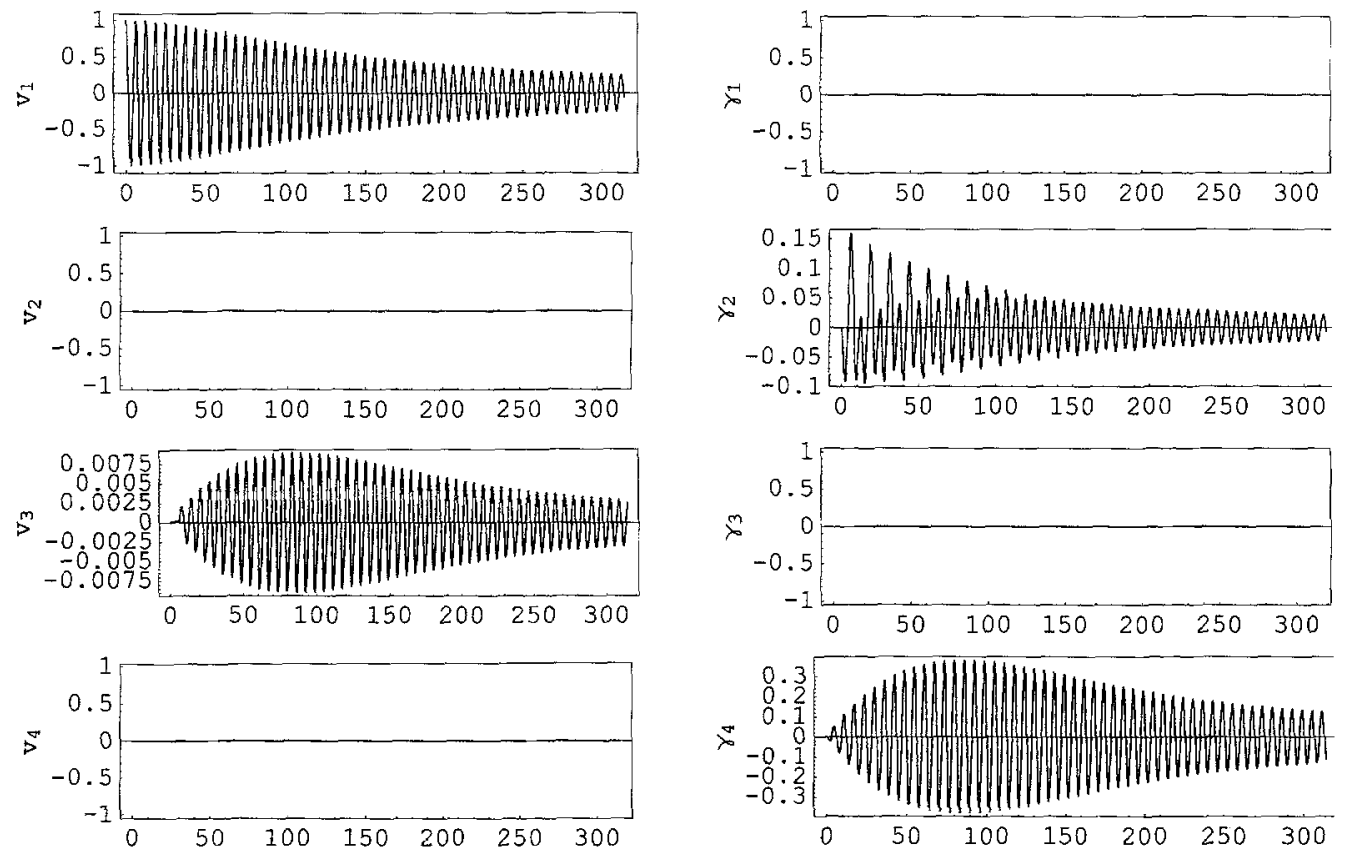

Fig. 8. Time evolution simulation ( $h=h_{14}^{*}$, dissipative line)

Figure 7 shows numerically that the line inductance $h=h_{14}^{*}$ couples mainly mechanical mode 1 with electrical mode 4 . Comparing Fig. 7 with Fig. 5, we remark that the amplitudes of vibration of modes are modulated with different periods when different critical inductances are chosen: the amplitude period when $h=h_{14}^{*}$ is greater. This is a measure of time elapsed in the energy flow from mechanical to electrical form.

In Fig. $8, h=h_{14}^{*}$, and the line is assumed dissipative $(40 \Omega / \mathrm{m}$ is now the value of optimal line resistance). Comparison with Fig. 6 shows that the damping ratio is now smaller; this fact could be expected observing the shape of the veering region associated in Fig. 3 and the value of the coupling coefficient associated (1m-4e) in Fig. 4.

\section{Concluding remarks}

A one-dimensional microstructured continuum has been introduced to model beams coupled to electrical transmission lines by PZT actuators; we have found the evolutions equations for the electro-mechanical system considered in the case of linear constitutive relations; the spatial differential operator governing the problem has been partitioned according to the partition of the state vector into a mechanical and electrical part. This analysis led to the formulation of a necessary coupling condition between mechanical and electrical modes and to the statement of an internal resonance condition. These results were used in Sect. 4 in order to prove that bending waves can be damped and controlled also by means of standard second-order electric transmission lines of distributed piezoelectric actuators.

Moreover it has been proven that electro-mechanical coupling can lead to self-excitation of mechanical modes also in linear systems. 


\section{References}

[1] Eringen, A. C., Maugin, G. A.: Electrodynamics of continua I-II. New York: Springer 1990.

[2] Fuller, C. R., Elliott, S. J., Nelson, P. A.: Active control of vibration. London: Academic Press 1996.

[3] Guran, A., Inman, D. J.: Wave motion, intelligent structures and nonlinear mechanics. Singapore: World Scientific 1995.

[4] Proceedings of SPIE: Smart materials and structures, Vol. 3241.

[5] dell'Isola, F., Vidoli, S.: Continuum modelling of piezoelectro-mechanical truss beams: an application to vibration damping. Arch. Appl. Mech. 68, 1-19 (1998).

[6] dell'Isola, F., Vidoli, S.: Damping of bending-waves in truss beams by electrical transmission line with PZT actuators. Arch. Appl. Mech. 68, 626-636 (1997).

[7] Di Carlo, A.: A non-standard format for continuum mechanics. In: Contemporary research in the mechanics and mathematics of materials (Batra, R. C., Beatty, M. F., eds.), pp. 263-268. CIMNE: Barcelona 1996.

[8] Maugin, G. A.: The method of virtual power in continuum mechanics: application to coupled fields. Acta Mech. 35, 1-70 (1980).

[9] Capriz, G.: Continua with microstructure. New York: Springer 1989.

[10] Euler, L.: Methodus inveniendi lineas curvas maximi minimive proprietati gaudentes. Lausanne (1744). German Translation in: Opera Omnia I (24), Berna (1952).

Bernoulli, J.: Specimen alterum calculi differentialis ... una cum additamento quodam ad problema funicularum, aliisque. Acta Eruditorum (1691).

[11] Di Carlo, A.: Bodies with affine structure. Lecture held at the 31st Polish Solid Mechanics Conference. Mierki, Sept. 1996.

[12] Gurtin, M. E.: An introduction to continuum mechanics. New York: Academic Press 1981.

[13] Chua, L. O., Desoer, C. A., Kuh, E. S.: Linear and nonlinear circuits. New York: McGraw-Hill 1987.

[14] Reed, M., Simon, B.: Methods of modern mathematical physics. Boston: Academic Press 1980.

[15] Krasnov, M. L., Kiselev, A. I., Makarenko, G. I.: Equazioni integrali. Moscow: Mir 1976.

Authors' address: S. Vidoli and F. dell'Isola, Dipartimento di Ingegneria Strutturale e Geotecnica, Università di Roma "La Sapienza", Via Eudossiana 18, I-00184 Roma, Italia 